\title{
Foreword by Guest Editors for the Special Issue on the 2011 ICUFN Conference
}

\author{
Zary Segall · Kyung Sup Kwak
}

C) Springer Science+Business Media, LLC. 2012

Welcome to this special issue of Wireless Personal Communications. This special issue constitutes a selection of best papers from the 2011 ICUFN (International Conference on Ubiquitous Future Networks) Conference. All these papers have been extended and reviewed again by 3 independent reviewers. ICUFN is an annual international conference (www.icufn. org) co-sponsored by IEEE Communications Society and organized by the KICS (Korean Information \& Communications Society).

The first paper is entitled "Joint Load Balancing of Radio and Transport Networks in LTE system". Load Balancing (LB) is an important mechanism often used in the cellular networks to offload the excessive traffic from high-load cells to low-load cells. In this paper, a novel concept of joint LB of transport network $(\mathrm{TN})$ and radio network $(\mathrm{RN})$ is proposed for LTE system based on a network-wide unified utility to capture the QoS of TN and RN. A simple local search algorithm is used for practical application. The proposed mechanism is shown by numerical results on both GBR and non-GBR traffic to have better performance than the conventional RN-only LB solution.

The second paper entitled "New QoS Resource Allocation Scheme Using GTS for WPANs" proposes a new GTS allocation scheme named unbalanced GTS allocation, which can improve the bandwidth resource efficiently. The network calculus theory based on the fluid model and greedy algorithm are applied to solve the bandwidth underutilization problem. Compared with the standard GTS allocation scheme, it shows an efficient bandwidth allocation with a QoS-guaranteed model and without breaking the standard protocol.

The third paper- "Power Allocation for Two-way relay System Based on Sequential Second Price Auction" proposes a sequential second price auction as a mechanism to

\footnotetext{
Z. Segall $(\varangle)$

Mobile Media and Services Lab, Royal Institute of Technology, Stockholm, Isafjordgatan 26, 4C, Kista 16440, Sweden

e-mail: zary@zary.com

K. S. Kwak

UWB Communications Research Center, School of Information and Communication Engineering, Inha University, 253 Yonghyun-Dong, Nam-Gu, Incheon, Korea

e-mail:kskwak@inha.ac.kr
} 
allocate the power of a two-way relay system with multiple user pairs. It is shown that the total channel capacity increases with the number of user pairs and the power units, and the system efficiency of this mechanism is high and stable.

The fourth paper entitled "Cross-layer Analysis of CSMA/iCA based Wireless Local Area Network" provides a cross layer analysis of the CSMA/iCA based WLAN and develops 3D Markov chain that emulates the backoff procedure of the CSMA/iCA. It is shown that CSMA/iCA offers better performance than CSMA/CA in the effects of channel noise and packet arrival rate at contending stations.

The fifth paper- "On the Design of Inclusive Ubiquitous Access" studies an affordable, high-performance, low-effect router based on open source software and standard off-theshelf, hardware offering both copper and fiber links. They discuss the design of key network elements and careful power management based on alternative energy sources and storage. Their design is capable of forwarding more than $700 \mathrm{kbps}$ at $22 \mathrm{~W}$. The power consumption is considerably less than all alternatives. This paper also reported on ongoing field-tests based on the use of the current version of this router for provisioning of broadband network services.

The final one entitled "Efficient utilization of Available Channels in Dynamic Spectrum Access Networks" proposes an error-adaptive MAC protocol by switching between errorrecovery mode and dual-transmit mode according to the channel status. When the channel is not good, additional channels acquired from the legacy network are used for the frame error recovery. On the other hand, in case that the channel is stable and reliable, the extra channels can be used to increase the throughput of cognitive devices. The simulation shows that the protocol can enhance the throughput of the cognitive radio networks.

I would like to thank the Editor-in-chief (Professor Ramjee Prasad) and Springer's senior editorial assistant (Mrs. Christine Velarde) for their support and help in realizing this special issue. Special thanks to all the authors for promptly revising their papers to the requirements of their viewers. Thanks to the organizers of ICUFN conference, especially to the Organizing Co-Chair (Professor Yeong Min Jang) and TPC Co-Chair (Professor Seung-Ho Jeong) for gathering quality papers for this special issue. I hope you will enjoy reading this special issue.

\section{Guest Editors}

Zary Segall and Kyung Sup Kwak

\section{Author Biographies}

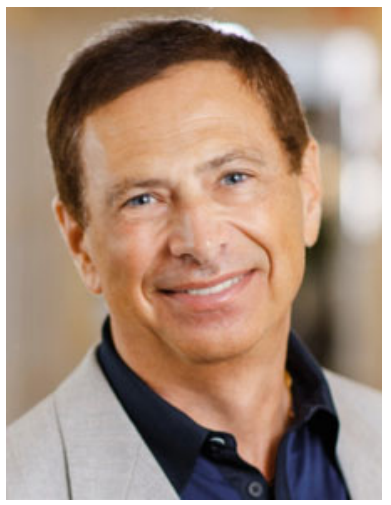

Zary Segall is currently Chair Professor in Scalable Mobile Services at The Royal Institute of Technology- KTH-and the Director of the Mobile Media and Service Lab, Stockholm, Sweden. As part of his research activity, he has developed theoretical methods and practical systems for highly dependable systems, fault tolerance, networking, wearable information systems, human aware computing and convergent design. Those systems have been conducive to software licensing to IBM, AT\&T, GE and NASA and to deployed systems by NASA missions, Air Traffic Control, Microsoft Operating Systems, telecommunication services, techno-fashion, wearable human aware artifacts and innovation processes. His research funding record include research grants and contracts from NSF, DARPA, ONR, GE, GM, Intel, Philips, GM, NASA, AT\&T, IBM, TeliaSonera, Ericsson, Vinnova, EU and The Next Generation Swedish Strategic Fund. For his education and research contribution he has been recognized as a Fellow of the IEEE Computer Society and as a Fulbright Distinguished IT Chair. Dr. Segall is the recipient of a number of national and international outstanding contribution awards. Prior to joining the Royal Institute of Technology in Sweden he was a Distinguished Professor in Computer Science and Engi- 
neering at University of Maryland (UMBC), Director of the UMBC Center for Convergent Design, Professor and Head of the Computer and Information Science Department at University of Oregon and Professor of Computer Science and Engineering at Carnegie Mellon University.

Kyung Sup Kwak received the B.S. degree from the Inha University, Inchon, Korea in 1977, and the M.S. degree from the University of Southern California in 1981 and the Ph.D. degree from the University of California at San Diego in 1988, under the Inha University Fellowship and the Korea Electric Association Abroad Scholarship Grants, respectively. From 1988 to 1989 he was with Hughes Network Systems, San Diego, California. From 1989 to 1990 he was with the IBM Network Analysis Center at Research Triangle Park, North Carolina. Since then he has been with the School of Information and Communication Engineering, Inha University, Korea as a professor. He had been the chairman of the School of Electrical and Computer Engineering from 1999 to 2000 and the dean of the Graduate School of Information Technology and Telecommunications from 2001 to 2002 at the Inha University, Inchon, Korea. He has been the directors of UWB Wireless Communications Research Center, a formerly key national IT research center, Korea. Since 1994 he served as the president of Korean Institute of Communication Sciences (KICS) in 2006, and the president of Korea Institute of Intelligent Transport Systems (KITS) in 2009. In 1993, he received Engineering College Achievement Award from Inha University, and a distinguished service medal from the Institute of Electronics Engineers of Korea (IEEK) in 1996, and in 1999 he received distinguished service medals from the KICS. He received the LG Paper Award in 1998, and Motorola Paper Award in 2000. He received Awards of research achievements on UWB radio technology from Minister of Information \& Communication, Prime Minister, and President of Korea in 2005 and 2006, respectively. Haedong Paper Award in 2007 and Haedong Prize of research achievement in 2009. In 2008, he was elected for Inha Fellow Professor (IFP). 